\title{
Correlation of the scattering and dispersion events in the Crab Nebula pulsar
}

\author{
A. Kuzmin ${ }^{1}$, B. Ya. Losovsky ${ }^{1}$, C. A. Jordan ${ }^{2}$, and F. G. Smith ${ }^{2}$ \\ 1 Pushchino Radio Astronomy Observatory of Lebedev Physical Institute, Russia \\ 2 University of Manchester, Jodrell Bank Observatory, Macclesfield, Cheshire, SK11 9DL, UK \\ e-mail: fgs@jb.man.ac.uk
}

Received 7 December 2007 / Accepted 23 February 2008

\section{ABSTRACT}

\begin{abstract}
In separate series of observations of the Crab pulsar, pulse broadening due to scattering was measured at $111 \mathrm{MHz}$, and variations of dispersion due to pulse delay were measured at higher radio frequencies. In a remarkable event lasting 200 days a large increase occurred in both parameters and with similar time signatures. The increases in scattering and dispersion measure observed over the 200 days MJD 53 950-54 150 are attributable to the effects of an ionised cloud or filament crossing the line of sight. The cloud would be $10^{11}-10^{12} \mathrm{~m}$ across, with electron density $10^{3}-10^{4} \mathrm{~cm}^{-3}$. The increased scattering might originate within the cloud itself, or the moving filament might induce turbulence in a separate higher density cloud in the line of sight.
\end{abstract}

Key words. pulsars: general - pulsars: individual: Crab - ISM: supernova remnants

\section{Introduction}

It has been known for many years that radio pulses from the Crab pulsar are affected both by a variable delay due to changes in dispersion and by a variable pulse broadening due to scattering along the line of sight (Rankin \& Counselman 1973; Isaacman \& Rankin 1977). Both phenomena vary on a typical time scale of about 100 days, but in previous observations their variations have appeared to be imperfectly correlated and possibly even uncorrelated. At our two observatories, we have maintained for several years two series of observations to separately monitor these two phenomena, and can now report a discrete event that shows a remarkably good correlation between variations in scattering and in dispersion measure.

\section{Observations}

Observations of dispersion measure are made at least once a week at Jodrell Bank Observatory as part of the Crab pulsar timing ephemeris which has been produced and made generally available since 1982 . The ephemeris is based on daily observations of time of arrival of pulses at $610 \mathrm{MHz}$, while the dispersion delay is measured by comparison with similar observations at $1400 \mathrm{MHz}$. Observations at Pushchino Radio Astronomy Observatory monitoring the pulse shape at $111 \mathrm{MHz}$ have continued since 2004. Both before and during the event the pulse is broadened with a steep rise and an approximately exponential decay with a time constant of several milliseconds; this characteristic decay time is monitored almost daily.

A distinctive property of the Crab pulsar low frequency observations is that the scatter broadening may be comparable with or greater than the pulsar period. To avoid the resulting confusion we use for observations the giant pulses of this pulsar, which stand out of the regular pulses as rare, strong, well defined single pulses. The pulse broadening is measured by fitting the convolution of a Gaussian template pulse with a truncated exponent as the thin screen scatter function, to the observed pulsar pulse.

The results of these measurements over a period of 600 days are shown in Fig. 1. This shows a discrete event, lasting 200 days (MJD 53 950-54 150), during which the dispersion and scattering changed together. The two curves are shown as recorded; note especially the sharp rise at the start of the event, and the delay of 30 days between the onset of the rise in scattering and the rise in DM. Both before and after this event there are smaller variations which are less obviously correlated. The event appears as a distinct phenomenon which stands out from the general level of variation in both parameters.

The dispersion measure is proportional to the total electron content along the line of sight. Most of this is attributed to the interstellar medium, and this component is not expected to show such large and rapid variations: observations of other pulsars show only comparatively small and slow variations, as shown by You et al. (2007). The base level of the dispersion measure appears to be $56.745 \mathrm{~cm}^{-3} \mathrm{pc}$; the event increases this by $\Delta \mathrm{DM} \approx$ $0.03 \mathrm{~cm}^{-3} \mathrm{pc}$. The observed scattering, by contrast, is more than doubled at the event, increasing from 10 to $25 \mathrm{~ms}$. Scattering by random variations in refractive index depends on irregular fluctuations of electron density within any part of the propagation path; the simplest interpretation is that the increased scattering and the increased dispersion are both due to a discrete electron cloud or filament within the Nebula.

\section{Size and location}

Three length scales are involved in estimating the size of a single cloud responsible both for increased dispersion and scattering:

1. The overall size $R$ of the cloud, which we will estimate from the duration of the event and the range of velocities which might be involved. We will assume that the thickness is also 


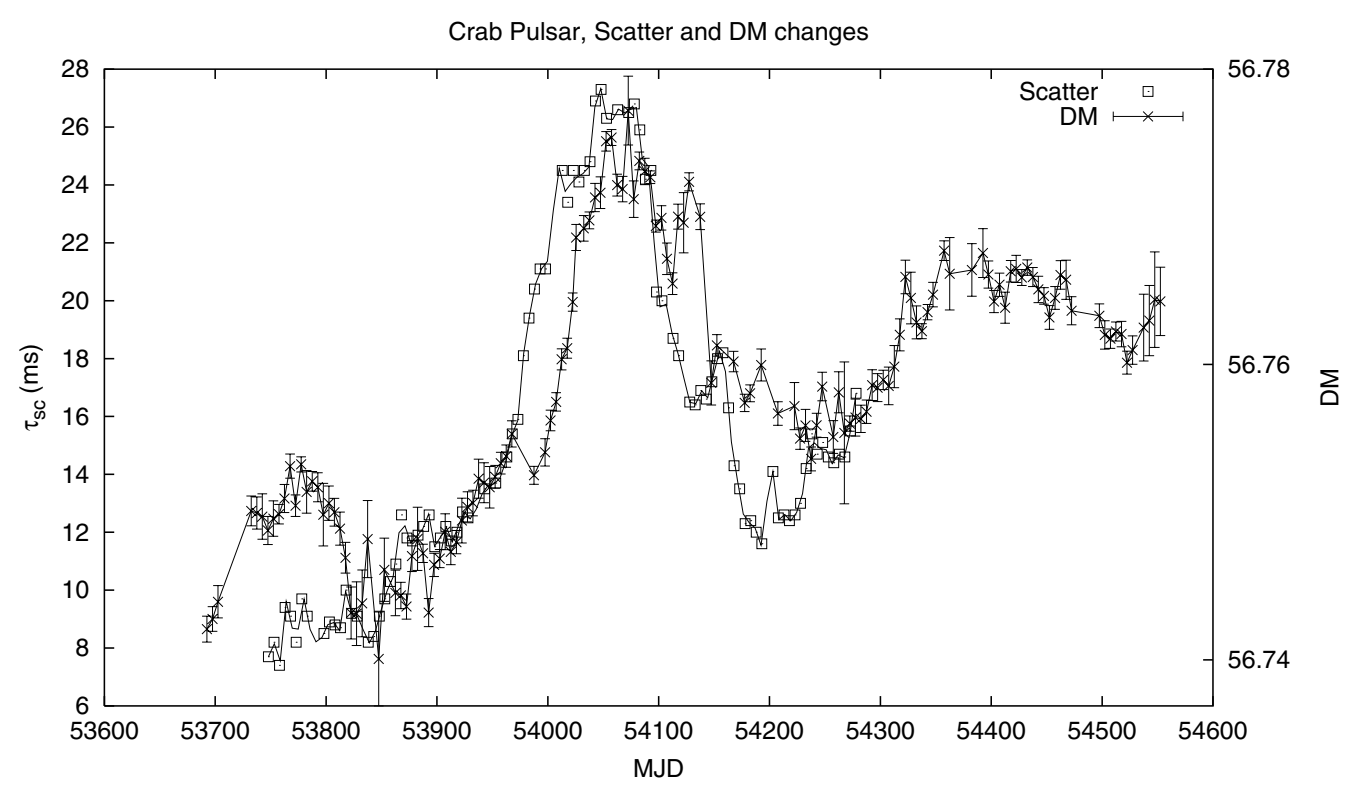

Fig. 1. Pulse scatter length at $111 \mathrm{MHz}$, and dispersion measure DM.

$R$. A maximum overall size may be estimated by assuming the velocity of the cloud across the line of sight does not exceed $100 \mathrm{~km} \mathrm{~s}^{-1}$, which for a duration of 200 days gives a maximum dimension $R \leq 2 \times 10^{12} \mathrm{~m}\left(=7 \times 10^{-5} \mathrm{pc}\right)$.

2. The distance $L$ from the pulsar to the cloud, which may be related to the observed wisps close to the pulsar (Bietenholz et al. 2004), i.e. $L \approx 0.1 \mathrm{pc}$, or it may be related to structure at the outer edge of the Crab Nebula (Sankrit et al. 1998), i.e. $L \approx 1.5 \mathrm{pc}$.

3. Scattering through the cloud appears as a disc whose radius $r$ is related to the pulse broadening. This disc must be smaller than the cloud itself; it must however be not much smaller, since scattering was observed before the cloud was in the direct line of sight. Since the observer is at a large distance compared with $L$, the pulse broadening $\tau$ is simply related to the scattering angle $\theta_{\mathrm{rms}}$ by $\tau=L \theta_{\mathrm{rms}}^{2} / 2 c$, giving a scattering angle of 8 arcsec for $L=0.1 \mathrm{pc}$ and $2 \operatorname{arcsec}$ for $L=1.5 \mathrm{pc}$. The radius of the scattering disc is $L \theta_{\mathrm{rms}}$, which is $10^{11} \mathrm{~m}$ for $L=0.1 \mathrm{pc}$ and $4.5 \times 10^{11} \mathrm{~m}$ for $L=1.5 \mathrm{pc}$. The cloud must therefore have a minimum dimension $R \geq 10^{11} \mathrm{~m}$.

Given these limits on $R$, and assuming the cloud has the same dimension along the line of sight, the observed change $\triangle \mathrm{DM}$ in dispersion measure gives an electron density $n_{\mathrm{e}}=$ $10^{3}-10^{4} \mathrm{~cm}^{-3}$. This high density is a strong indication that the cloud is within the Nebula, and probably associated with activity near the centre of the Nebula. We note also that Rankin et al. (1988) observed a change in Faraday rotation associated with a similar change in dispersion, which they interpret as a magnetic field value of $160 \mu \mathrm{G}$ within a filament of ionised gas. These events may be related to a larger and more complex pulse scattering event observed in 1997-8 (Lyne et al. 2001).

The sharp onset of both scattering and increased DM indicate that the leading edge of the cloud is well-defined, with a maximum scale of about $3 \times 10^{10} \mathrm{~m}$. The earlier onset of scattering is consistent with our model, since scattering is expected to be observable before the direct line of sight is crossed by the cloud.
Other interpretations of this event, involving more than one component of the Nebula, are of course possible. In particular, we note that Kuzmin et al. (2008) have shown that a pulse time delay observed at low frequencies, additional to the frequency $v^{-2}$ dependence, indicates the existence of a region in the line of sight with an electron density as high as $10^{6} \mathrm{~cm}^{-3}$. Turbulence in the moving cloud which we now observe may have been induced by a collision with such a dense region.

\section{Summary}

The increases in scattering and dispersion measure observed over the 200 days MJD 53950-54150 are attributable to the effects of an ionised cloud or filament crossing the line of sight. The cloud would be $10^{11}-10^{12} \mathrm{~m}$ across, with electron density $10^{3}-10^{4} \mathrm{~cm}^{-3}$. The increase in scattering might be due to the cloud itself, or to turbulence induced in a high electron density cloud in the line of sight.

Acknowledgements. Research at the Pushchino Radio Astronomy Observatory was partly supported by Russian Foundation for Basic Research N 05-02-16415. A. Kuzmin and B. Losovsky thank A. Aleksandrov, V. Ivanova, I. I. Litvinov, S. Logvinenko, T. Semina and M. Tchereshnev for assistance in observations and data reduction.

\section{References}

Bietenholz, M. F., Hester, J. J., Frail, D. A., \& Bartel, N. 2004, ApJ, 615, 794 Isaacman, R., \& Rankin, J. M. 1977, ApJ, 214, 214

Kuzmin, A. D., Losovsky, B. Y., Logvinenko, S. V., \& Litvinov, I. I. 2008, Astron. Lett., submitted

Lyne, A. G., Pritchard, R. S., \& Graham-Smith, F. 2001, MNRAS, 321, 67

Rankin, J. M., \& Counselman, C. C. 1973, ApJ, 181, 875

Rankin, J. M., Campbell, D. B., Isaacman, R. B., \& Payne, R. R. 1988, A\&A, 202, 166

Sankrit, R., Hester, J. J., Scowen, P. A., et al. 1998, ApJ, 504, 344

You, X. P., Hobbs, G., Coles, W. A., et al. 2007, MNRAS, 378, 493 